\title{
From Development to Implementation: Adjusting the Hematocrit of Deglycerolized Red Cell Concentrates to Meet Regulatory Standards
}

\author{
Tracey Turner Adele Hansen Jayme Kurach Jason P. Acker \\ Canadian Blood Services, Centre for Innovation, Edmonton, AB, Canada
}

\section{Keywords}

Red blood cells . Hematocrit - COBE 2991 .

Glycerolization · Deglycerolization

\section{Summary}

Background: Before transfusion, thawed frozen red cell concentrates (RCCs) must be deglycerolized. In order to ensure that these products meet regulatory standards for hematocrit, an approach to manipulate hematocrit post deglycerolization was developed and implemented. Methods: Glycerolized and frozen RCCs were thawed and deglycerolized using the COBE 2991 cell processor, and the final product's hematocrit was adjusted by addition of various volumes of $0.9 \%$ saline / $0.2 \%$ dextrose. The in vitro quality of RCCs (hematocrit, hemolysis, hemoglobin content, volume, recovery, ATP, supernatant potassium, and others) were compared to Canadian Standards Association (CSA) and other standards for deglycerolized RCCs. Results: Addition of saline/dextrose re-suspension solution in a range of $65-90 \mathrm{~g}$ post deglycerolization led to acceptable hematocrits. In the pilot study, this approach resulted in RCCs meeting all CSA standards for deglycerolized RCCs, with stimulation of RBC metabolism demonstrated by increased ATP concentration. In the validation phase, results were similar, although the CSA hemolysis standard was not met. Preand post-implementation data confirmed that manipulated RCCs met CSA hematocrit standards. Conclusion: This process was implemented at Canadian Blood Services to provide deglycerolized RCCs that meet the CSA hematocrit standard. However, pre- and post-implementation data reveal that this deglycerolization process is not sufficient to have RCCs consistently meet hemolysis standards.

(C) 2016 S. Karger GmbH, Freiburg

\section{Introduction}

Red cell concentrates (RCCs) that are stored refrigerated for up to 42 days are used routinely in most red blood cell (RBC) transfusions. Cryopreservation (freezing) can extend RCC storage time to 10 years and beyond $[1,2]$, and is predominantly used to preserve stocks of rare blood phenotypes, for storage for autologous and allogeneic use, and for military applications [3]. Cryopreservation is possible through the addition of high concentrations of glycerol, which acts as a cryoprotectant, to the RCC before freezing [3]. In North America, RCCs are commonly cryopreserved in $40 \% \mathrm{w} / \mathrm{v}$ glycerol concentrations with slow cooling rates $\left(\sim 1{ }^{\circ} \mathrm{C} / \mathrm{min}\right)$ prior to storage at $\leq-65{ }^{\circ} \mathrm{C}$, while in Europe lower glycerol concentrations and rapid freezing are also used [4]. After thawing cryopreserved RCCs, the glycerol must be removed. The process of deglycerolization is complicated and time-consuming, but is necessary before the unit can be transfused to avoid intravascular hemolysis of the RBCs and subsequent post-transfusion adverse events.

The current method used by Canadian Blood Services to deglycerolize RCCs employs the COBE 2991 cell processor (TerumoBCT), an open system that produces products with an outdate of $24 \mathrm{~h}$. Deglycerolization is achieved using multiple washes with saline solutions of decreasing concentrations. Each wash is followed by centrifugation and removal of the supernatant, a process that is automatically stopped when RBCs are detected in the exit tubing to the waste bag. This results in an RCC that is more concentrated and has a lower supernatant volume and thus a higher hematocrit (Hct) than a standard RCC processed from whole blood (WB) that has not undergone deglycerolization. Past quality control (QC) data at Canadian Blood Services indicate that the average Hct of deglycerolized RBC units is $0.81 \pm 0.04 \mathrm{l} / \mathrm{l}$ (range 0.74 $0.931 / 1, n=75)$. Thus, $53 \%$ of deglycerolized RBC units tested for QC do not meet regulatory requirements for Hct (Canadian Standards Association (CSA) standard states that Hct must be $\leq 0.8 \mathrm{l} / \mathrm{l}$ in $90 \%$ of units tested [5]). In other jurisdictions, the Council of Eu-

\section{KARGER}

() 2017 S. Karger GmbH, Freiburg
Jason P. Acker, MBA, PhD Canadian Blood Services Centre for Innovation 8249-114 Street, Edmonton, AB T6G 2R8, Canada jason.acker@blood.ca 
rope recommends that deglycerolized RCCs have a Hct of 0.35 $0.701 / 1$ [6], while the AABB (2009) guidelines do not state a specific Hct recommendation for deglycerolized RCCs but generally recommend a Hct of $\leq 0.80 \mathrm{l} / \mathrm{l}$ for RBC units [7].

In this study, we developed and investigated an approach to manipulate RCCs post processing on the COBE 2991 in order to ensure that these products meet the Hct regulatory standard. The approach involved addition of saline/dextrose to the deglycerolized RCC and was designed to have as little impact on the standard operating procedure as possible. The hypothesis was that addition of solution would reduce the RCCs' Hct and result in products with Hct within the acceptable range. Here we report the results of the study to investigate and validate this approach and determine its impact 1-year post implementation.

\section{Material and Methods}

\section{Pilot Study}

\section{Estimation of Re-Suspension Solution Volumes}

For standard RCCs produced by Canadian Blood Services that are stored refrigerated and do not undergo freezing/deglycerolization, the Canadian Blood Services Circular of Information (2011) indicates Hct should be $0.5-0.71 / 1$ in at least $90 \%$ of units [8]. Therefore, an ideal Hct value for deglycerolized RCCs post processing of $0.60 \mathrm{l} / \mathrm{l}$ was chosen. However, as manual addition of re-suspension solution was chosen to manipulate deglycerolized RCCs post processing, it was decided a range of target Hct values would best accommodate this process. These values were used to calculate the volume of re-suspension solution needed to adjust the Hct to a range of acceptable values $(0.501 / 1,0.601 / 1$, and $0.70 \mathrm{l} / \mathrm{l})$ as follows: $(0.81 \mathrm{l} / 1 \times 224 \mathrm{ml})$ divided by the desired Hct. The average $\mathrm{QC}$ volume was then subtracted from the result to determine the volume of re-suspension fluid needed to adjust to the desired Hct. Re-suspension solution was added by weight; therefore, the volumes calculated were converted to gram. An estimated addition of $65 \mathrm{~g}, 75 \mathrm{~g}$ and $90 \mathrm{~g}$ of re-suspension solution would result in a theoretical average Hct of $0.701 / 1,0.601 / 1$ and $0.501 / 1$, respectively.

\section{RCC Collection and Processing}

This was a pool-and-split study using RCCs processed by both methods used at Canadian Blood Services. Briefly, WB $(\mathrm{n}=48)$ was collected from healthy volunteers into either MacoPharma (Tourcoing, France) or Fenwal (Lake Zurich, IL, USA) collection sets with citrate-phosphate-dextrose (CPD) anticoagulant. 24 of the units were processed using the buffy coat method, as described previously [9]. The remaining 24 were processed by the WB filtration method, also as described previously [9]. From these, 12 groups of 4 units each, matched for processing method, collection date and ABO group, were pooled into large 21 Fenwal bags. Pools were mixed and split into equal volumes $( \pm 10 \mathrm{~g}$ of each other) back into their original bags. RCCs were weighed using a GSE 574 scale (GSE Scale Systems, Livonia, MI, USA) and stored in a refrigerator at $1-6{ }^{\circ} \mathrm{C}$ until glycerolization and freezing.

\section{RCC Glycerolization and Freezing}

The RCC, at 14 days post collection, and glycerol solution (Glycerolyte 57 Solution - 4A7831; Baxter Health Care Corporation, Deerfield, IL, USA) were warmed in a $37^{\circ} \mathrm{C}$ water bath for $20-30 \mathrm{~min}$ and then attached to a 21 freezing kit (4R2422, Fenwal, Lake Zurich, IL, USA). The glycerol solution was added using a two-step method: $100 \mathrm{ml}$ was added with gentle mixing followed by an equilibration period of 5-15 min. An additional $300 \mathrm{ml}$ was added to achieve an approximately $40 \%$ glycerol concentration in the RCC. The glycerolized RCC was placed into a metal freezing cassette and stored in a $-80{ }^{\circ} \mathrm{C}$ freezer for 24-48 h.
Thawing of Frozen RCCs and Deglycerolization

Frozen RCCs were placed in a $37^{\circ} \mathrm{C}$ water bath for 7-15 min until thawed. The RCC was attached to a COBE 2991 Blood Cell Processing Set (REF90819, TerumoBCT, Lakewood, CO, USA) and loaded onto the COBE 2991 Blood Cell Processor following the manufacturer's instructions. Before automated washing, $150 \mathrm{ml}$ of $12 \%$ sodium chloride processing solution (4B7874Q, Baxter Health Care Corporation) was manually added to the thawed RCC over $60 \mathrm{~s}$ with constant mixing. The RCC was allowed to equilibrate for 3-5 min before the addition, with constant mixing, of approximately $650 \mathrm{ml}$ of $1.6 \%$ sodium chloride processing solution (4B7871, Baxter Health Care Corporation). The RCC was then processed using the automated settings on the COBE 2991 which involved one additional wash with $1.6 \%$ sodium chloride followed by two washes with $0.9 \%$ saline / $0.2 \%$ dextrose.

During the final centrifugation of the washed RCC, a visual supernatant hemoglobin $(\mathrm{Hb})$ reading was obtained using a color comparator (\#18501C; Haemonetics, Braintree, MA, USA). If the visual supernatant $\mathrm{Hb}$ level was graded $>4$ (on a scale of 1-8), an additional wash with $1,000 \mathrm{ml}$ of $0.9 \%$ sodium chloride (JB1324, Baxter Health Care Corporation) was performed to reduce the supernatant $\mathrm{Hb}$ concentration. A new bag of $0.9 \%$ saline / $0.2 \%$ dextrose was then attached and used as the re-suspension fluid for the RBCs. The tubing between the RCC and the re-suspension solution was clamped using a hemostat, and the processing kit was removed from the processor (including the lid of the centrifuge).

\section{Addition of Re-Suspension Solution}

The RCC and the COBE 2991 centrifuge lid were placed onto a GSE 574 scale (GSE Scale Systems), and the scale was tarred. The $0.9 \%$ saline / $0.2 \%$ dextrose re-suspension solution was hung at least $90 \mathrm{~cm}$ above the RCC. Each of the four units in an RCC pool received a different mass of re-suspension solution: $0 \mathrm{~g}$ (Group A; control), $65 \mathrm{~g}$ (Group B), $75 \mathrm{~g}$ (Group C) or $90 \mathrm{~g}$ (Group D), all $\pm 5 \mathrm{~g}$. All RCCs were stored in a $1-6^{\circ} \mathrm{C}$ refrigerator.

\section{RCC Sampling}

RCCs were gently mixed and inverted five times. Samples were obtained by inserting an 18-gauge needle attached to a syringe through a sampling site coupler (4C2405, Fenwal) inserted into one of the unit ports. The needle was removed, and the sample was dispensed drop-wise into aliquot tubes for testing. RCCs were sampled at three different time points during the study; $3 \mathrm{ml}$ prior to glycerolization, $1 \mathrm{ml}$ immediately post deglycerolization for spun hct, and 10 $\mathrm{ml}$ at 20-24 h after re-suspension post deglycerolization for measurement of RBC parameters.

\section{RCC and RBC Parameters}

Mean cell volume (MCV), Hb, and Hct were measured on an automated Advia 120 Hematology Analyzer (Siemens, Mississauga, ON, Canada). Spun Hct, ATP concentration, supernatant potassium, Drabkin's total and supernatant $\mathrm{Hb}$, and RBC hemolysis were determined as described previously [9].

Due to the differences in dilutions between treatment groups, supernatant potassium was corrected using the following calculation as described previously [11]:

Supernatant potassium $(\mu \mathrm{mol} / \mathrm{gHb})=($ supernatant potassium $(\mathrm{mmol} / \mathrm{l}) /($ hemoglobin $(\mathrm{g} / \mathrm{l}) \times 1,000$

Total $\mathrm{Hb}$ and supernatant $\mathrm{Hb}$ per unit were determined using the following calculations:

$\mathrm{Hb}(\mathrm{g} /$ unit $)=$ volume $(\mathrm{l}) \times \mathrm{Hb}(\mathrm{g} / \mathrm{l})$

Supernatant $\mathrm{Hb}(\mathrm{g} /$ unit $)=$ volume $(\mathrm{l}) \times(1-\mathrm{Hct}(\mathrm{h})) \times$ supernatant $\mathrm{Hb}(\mathrm{g} / \mathrm{l})$

Pre-glycerolization and post-deglycerolization mass were measured for each unit using a GSE 574 scale (GSE Scale Systems). RBC recovery was calculated using these masses and the Hct results obtained from the Advia 120 (Siemens) using the following calculation: 
Statistical Analysis

Differences between study groups were analyzed using an ANOVA with Tukey HSD post-hoc analysis (SPSS 23, IBM Corporation, Armonk, NY, USA). Results were considered significant when $\mathrm{p}$ values were less than 0.05 .

\section{Results}

Pilot Study

\section{CSA and Other Criteria}

Prior to glycerolization, no significant differences in volume $(300 \pm 22 \mathrm{ml}, 300 \pm 21 \mathrm{ml}, 300 \pm 22 \mathrm{ml}$ and $299 \pm 22 \mathrm{ml}$ in Groups $\mathrm{A}, \mathrm{B}, \mathrm{C}$ and $\mathrm{D}$, respectively) and Hct (0.652 $\pm 0.021 \mathrm{l} / \mathrm{l}, 0.655 \pm$ $0.018 \mathrm{l} / \mathrm{l}, 0.654 \pm 0.020 \mathrm{l} / \mathrm{l}$ and $0.654 \pm 0.018 \mathrm{l} / \mathrm{l}$ in Groups A, B, C and $\mathrm{D}$, respectively) were observed, indicating that the pooling and splitting steps taken were effective. Spun Hcts immediately post deglycerolization but prior to addition of the re-suspension solution were $0.80 \pm 0.02 \mathrm{l} / \mathrm{l}, 0.81 \pm 0.02 \mathrm{l} / \mathrm{l}, 0.81 \pm 0.02 \mathrm{l} / \mathrm{l}$ and $0.81 \pm 0.01 \mathrm{l} / \mathrm{l}$ in Groups A, B, C and D, respectively. In each group ( $\mathrm{n}=12$ RCCs), the mass of re-suspension solution added after deglycerolization was (mean $\pm \mathrm{SD}$ ): Group A/control - 0 g; Group B (low volume: target $65 \mathrm{ml}$ ) $-65 \pm 2 \mathrm{~g}$; Group C (medium volume: target 75 $\mathrm{ml})-75 \pm 2 \mathrm{~g}$; and Group D (high volume: target $90 \mathrm{ml}$ ) $-90 \pm 1 \mathrm{~g}$.

Confirming the premise of the study, control RCCs (Group A) failed to meet CSA criteria for Hct (table 1). The addition of resuspension solution reduced the Hct in a linear manner, and RCCs in Groups B, C, and D had statistically lower Hct compared with Group A and met the CSA Hct standards. RCCs from all experimental groups met the CSA standard for Hb content. All experimental groups met the CSA standard for hemolysis with $100 \%$ of units passing in Groups B, C, and D while only 92\% passed in Group A. Finally, all groups met the CSA and AABB standard for recovery, with Group A having statistically lower $\mathrm{RBC}$ recovery than Groups B, C and D; however, this difference may be related to the recovery calculations used.

Similarly, Group A RCCs failed to meet Council of Europe Hct guidelines for deglycerolized RCCs (table 1), while Group B, C, and D RCCs passed, and RCCs from all experimental groups met the Council of Europe standard for $\mathrm{Hb}$ content. Control units failed to meet Council of Europe guidelines for volume, but RCCs that had additional re-suspension solution added passed the standard and, as expected, had statistically higher volumes than control Group A. The Council of Europe standard for supernatant $\mathrm{Hb}$ in deglycerolized RCCs (g/unit) was not met by any group, and Group A was the only group in which any units passed the standard with a total of $25 \%$ passing the numerical standard.

Groups B, C, and D all fell within the average Hct range stated in the 2015 Canadian Blood Services Circular of Information for standard, liquid-stored RBCs that have not been frozen/deglycerolized (0.54-0.68 l/1) [14].

\section{Other RCC Quality Assessments}

RCCs in Groups B, C, and D had significantly higher ATP concentrations compared to Group A (fig. 1). There were lower super-
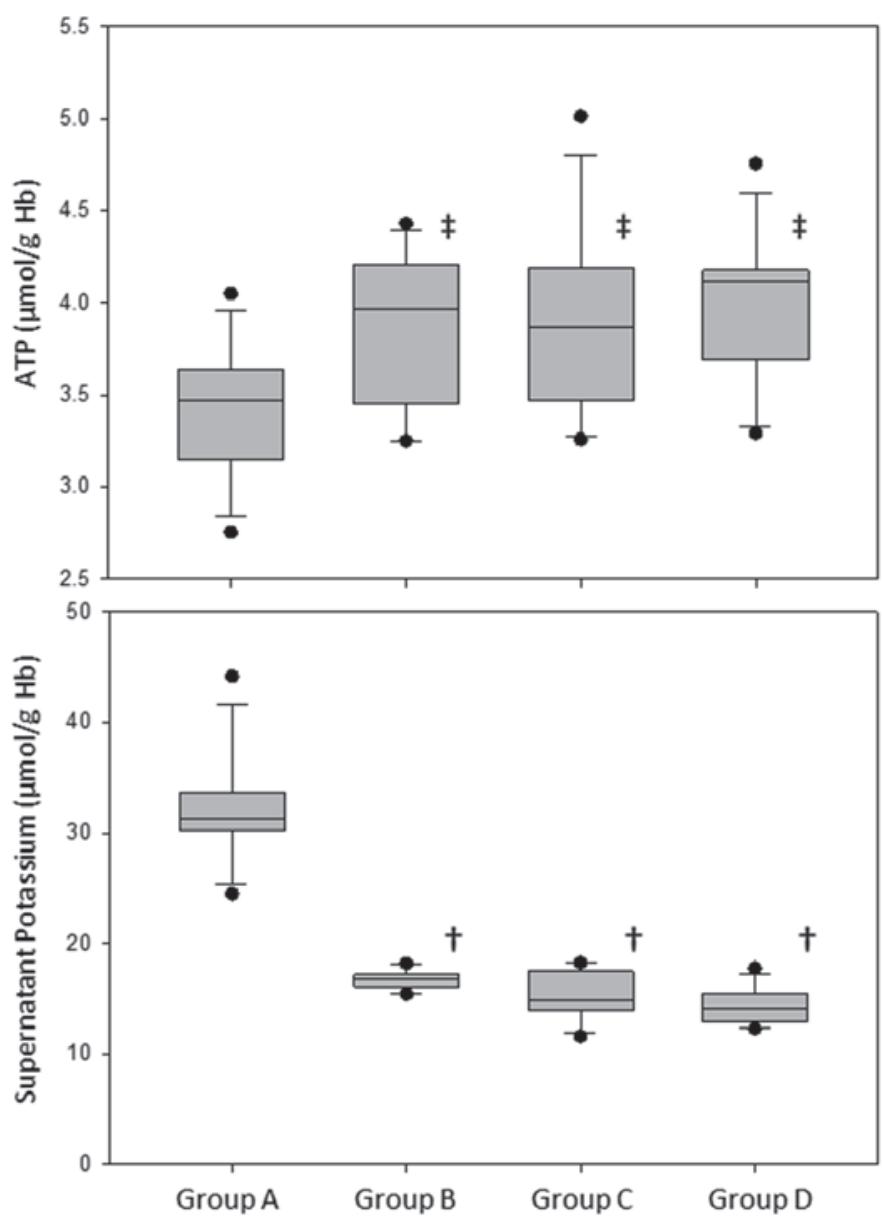

Fig. 1. ATP concentration and supernatant potassium in RCCs $20-24 \mathrm{~h}$ post deglycerolization (pilot study). Target volumes for additional re-suspension solution added after deglycerolization were as follows: Group A (control) - $0 \mathrm{ml}$; Group B (low volume) - $65 \mathrm{ml}$; Group C (high volume) - $75 \mathrm{ml}$; and Group D (medium volume) - $90 \mathrm{ml}$. ATP concentration was measured 20-24 h later using a commercial kit. $\mathrm{N}=12$ paired pooled and split RCC in each experimental group. Supernatant potassium was measured 20-24 h later using ion selective potentiometry. ${ }^{\dagger}$ Denotes statistically significant difference when compared to Group A ( $\mathrm{p}<0.001)$. ${ }^{\ddagger}$ Denotes statistically significant difference when compared to Group A $(\mathrm{p}<0.05)$.

natant potassium levels in the RCCs with extra re-suspension solution added, with Group A having significantly higher levels than all other groups (fig. 1). In all groups, there was a significant increase in MCV ( $\mathrm{p}<0.001$; data not shown) after deglycerolization, suggesting that swelling occurs with glycerolization/freezing/deglycerolization. Post deglycerolization, there were no differences among groups $\mathrm{A}, \mathrm{B}, \mathrm{C}$, and $\mathrm{D}$ for $\mathrm{MCV}$.

\section{Validation Study}

The actual volume of re-suspension solution added in the validation was $76 \pm 4 \mathrm{~g}$ (range $68-88 \mathrm{~g} ; \mathrm{n}=60$ ). The average age of the units prior to freezing was $13 \pm 1$ days, and the average length of 
Table 2. Validation study: 24- to 48 -hour post-deglycerolization RCC results compared to CSA [5], Council of Europe [6], and AABB [7] guidelines for deglycerolized RCCs ${ }^{\mathrm{a}}$

\begin{tabular}{|c|c|c|c|}
\hline & $\begin{array}{l}\text { All RCCs } \\
(\mathrm{n}=60)\end{array}$ & $\begin{array}{l}\text { Buffy coat } \\
\text { processed } \\
(\mathrm{n}=40)\end{array}$ & $\begin{array}{l}\text { Whole blood } \\
\text { processed } \\
(\mathrm{n}=20)\end{array}$ \\
\hline Hematocrit, $l / l$ & $0.61 \pm 0.03$ & $0.60 \pm 0.03$ & $0.61 \pm 0.03$ \\
\hline CSA & pass & pass & pass \\
\hline $90 \%$ of units $\leq 0.81 / 1$ (pass/fail) & (100\% pass) & (100\% pass) & (100\% pass) \\
\hline Council of Europe & pass & pass & pass \\
\hline $100 \%$ of units $0.35-0.701 / 1$ (pass/fail) & (100\% pass) & (100\% pass) & (100\% pass) \\
\hline Hemoglobin, $g / U$ & $48.1 \pm 5.2$ & $46.2 \pm 4.6$ & $51.9 \pm 4.2^{*}$ \\
\hline CSA & pass & pass & pass \\
\hline $90 \%$ of units $\geq 35 \mathrm{~g} / \mathrm{unit}$ (pass/fail) & (98\% pass) & (98\% pass) & (100\% pass) \\
\hline Council of Europe & fail & fail & pass \\
\hline $100 \%$ of units $\geq 36 \mathrm{~g} /$ unit (pass/fail) & (98\% pass) & (98\% pass) & (100\% pass) \\
\hline Volume, $m l$ & $273 \pm 25$ & $262 \pm 20$ & $297 \pm 16^{*}$ \\
\hline Council of Europe & pass & pass & pass \\
\hline $100 \%$ of units $>185 \mathrm{ml}$ (pass/fail) & (100\% pass) & (100\% pass) & (100\% pass) \\
\hline RBC hemolysis, \% & $0.73 \pm 0.28$ & $0.72 \pm 0.19$ & $0.76 \pm 0.22$ \\
\hline CSA & & & \\
\hline $90 \%$ of units $<0.8 \%$ (pass/fail) & (53\% pass) & (58\% pass) & (45\% pass) \\
\hline Supernatant hemoglobin, $g / U$ & $0.354 \pm 0.109$ & $0.335 \pm 0.102$ & $0.390 \pm 0.114$ \\
\hline Council of Europe & fail & & \\
\hline $100 \%$ of units $<0.2 \mathrm{~g} / \mathrm{U}$ (pass/fail) & (3\% pass) & (5\% pass) & (0\% pass) \\
\hline Recovery, \% & $92 \pm 5$ & $92 \pm 5$ & $93 \pm 5$ \\
\hline CSA and $\mathrm{AABB}$ & pass & pass & pass \\
\hline \multicolumn{4}{|l|}{ Mean $\mathrm{RBC}$ recovery in units tested $\geq 80 \%$ (pass/fail) } \\
\hline
\end{tabular}

frozen storage was $17 \pm 2$ days for buffy coat-processed RCCs and $9 \pm 4$ days for WB filtration-processed RCCs.

All units tested met both CSA and Council of Europe standards for Hct (table 2). All RCCs met the CSA standard for $\mathrm{Hb}$ content as well as CSA and AABB recovery standards for deglycerolized units. Contrary to the results of the pilot study, RCCs failed to meet CSA standards for RBC hemolysis. WB filtration-processed units were less likely to meet this standard than buffy coat-processed units. Buffy coat-prepared RCCs that required an additional saline wash during deglycerolization (based on a visual supernatant $\mathrm{Hb}$ reading) were even more likely to fail the CSA hemolysis standard. Both WB filtration-processed RCCs that underwent a second wash failed to meet the hemolysis standard.

The Council of Europe standard for Hb content requires 100\% of units to pass, but overall only $98 \%$ of the RCCs had Hb content greater than $36 \mathrm{~g} /$ unit (table 2). Buffy coat-processed RCCs had significantly lower $\mathrm{Hb}$ content than WB filtration-processed units $(\mathrm{p}<0.001)$ and failed to meet Council of Europe guidelines, while WB filtration-processed RCC did. One buffy coat-processed RCC that failed the Council of Europe standard (and those units that fell under $35 \mathrm{~g} /$ unit limit in the CSA standards) went through the additional wash process based on a visual supernatant $\mathrm{Hb}$ reading. Similar to the results of the pilot study, RCCs in the valida- tion study also failed the Council of Europe supernatant $\mathrm{Hb}$ standard.

\section{Pre- and Post-Implementation Quality Control Data Analysis}

Once implemented, an analysis of routine QC data collected on deglycerolized RCCs was undertaken. The new process yielded a deglycerolized RBC product that meets the CSA Hct standard in practice; post-implementation Hct was significantly lower than the pre-implementation Hct values (table 3; $\mathrm{p}<0.001$ ). In practice, the pre- and post-implementation $\mathrm{Hb}$ content per unit met the CSA standard and the Council of Europe standard. For volume, all units met the standard, but volume was significantly higher after implementation $(p<0.001)$. As observed in the validation study, in both the pre- and post-implementation groups the CSA standard for RBC hemolysis was not met, nor was the Council of Europe standard for supernatant $\mathrm{Hb}$. The pre-implementation data were aggregated from 10 buffy coat and $65 \mathrm{WB}$ filtration units where the postimplementation data were aggregated from 20 buffy coat and 49 WB filtration units. Pre implementation, $46 \%$ of buffy coat-produced units and $72 \%$ of WB filtration-produced units met the hemolysis standard. Post implementation, 50\% of buffy coat-produced units and $45 \%$ of WB filtration-produced units met the hemolysis standard. 
Table 3. Pre- and post-implementation study: 24- to 48-hour post-deglycerolization of RCC results compared to CSA [5], Council of Europe [6], and AABB [7] guidelines for deglycerolized RCCs ${ }^{\mathrm{a}}$

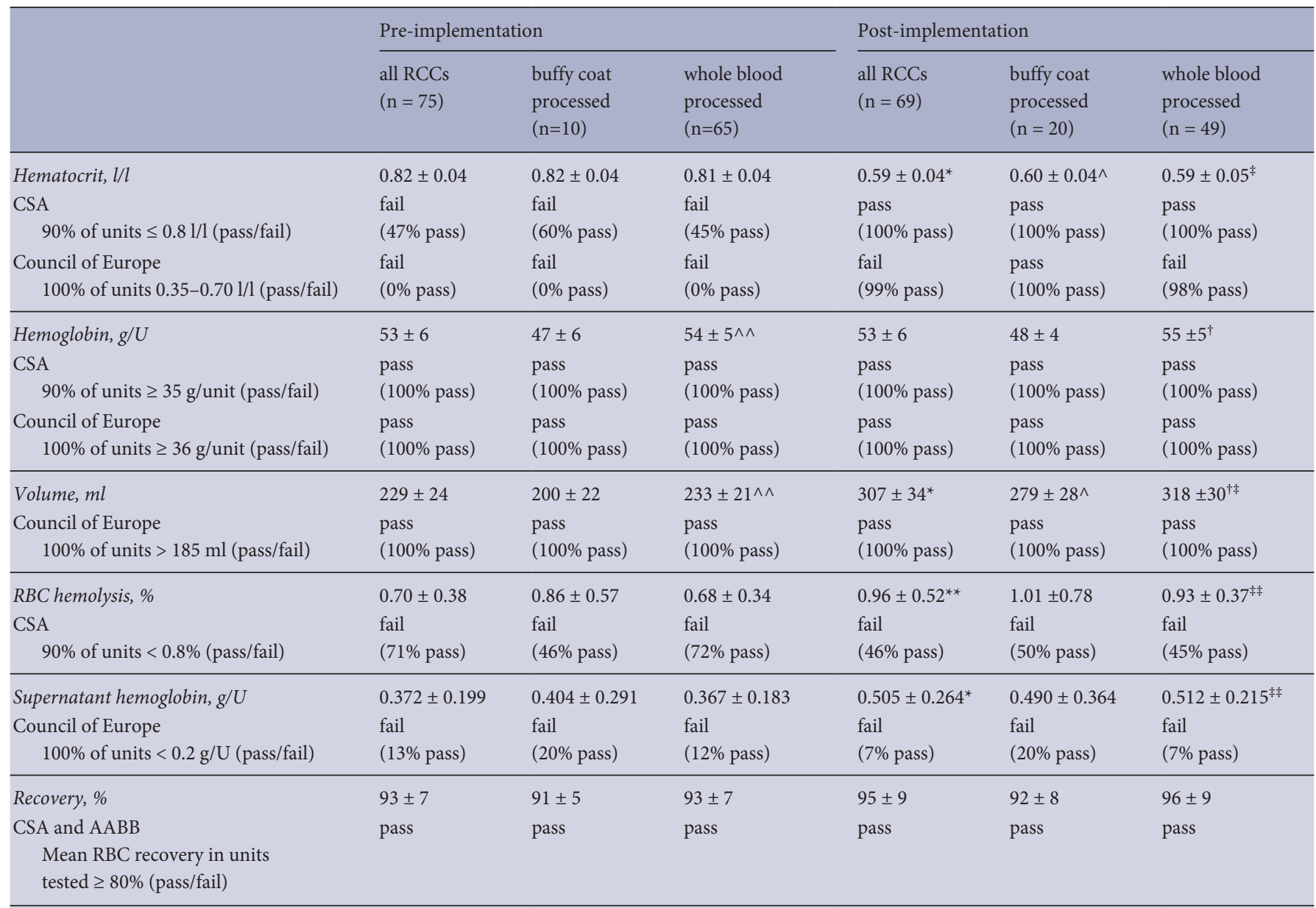

${ }^{a}$ Results are shown as mean \pm 1 SD.

* Statistically significant difference when compared to pre-implementation values $(\mathrm{p}<0.001)$.

${ }^{*}$ Statistically significant difference when compared to pre-implementation values $(\mathrm{p}<0.05)$.

$\wedge$ Statistically significant difference when compared to pre-implementation buffy coat processed $(\mathrm{p}<0.001)$.

$\wedge \wedge$ Statistically significant difference when compared to pre-implementation buffy coat processed $(\mathrm{p}<0.05)$.

†Statistically significant difference when compared to post-implementation buffy coat processed $(\mathrm{p}<0.001)$.

‡Statistically significant difference when compared to pre-implementation whole blood processed $(\mathrm{p}<0.001)$.

${ }^{\ddagger}$ Statistically significant difference when compared to pre-implementation whole blood processed $(\mathrm{p}<0.05)$.

\section{Discussion}

Hct reflects the packed RBC volume of a unit and is related to the RBC dose. CSA guidelines for Hct are broad, stating an upper limit of $0.80 \mathrm{l} / \mathrm{l}$. Beyond simply meeting the standard of less than $0.80 \mathrm{l} / \mathrm{l}$, we developed an approach that would result in deglycerolized RCCs with Hct levels that were comparable to the standard, liquid-stored RCCs produced at Canadian Blood Services (mean \pm 2 SD in 2011 Circular of Information (valid when study was initiated): $0.61 \pm 0.07$ 1/1 [8]). We also sought an approach that would have as little impact as possible on the already labor-intensive process of thawing and deglycerolizing frozen RCCs. The findings of the pilot study led to the recommendation that a target of 75 $\mathrm{g}$ (range of $65-90 \mathrm{~g}$ ) of $0.9 \%$ saline / $0.2 \%$ dextrose solution be used to re-suspend RCCs following deglycerolization employing the
COBE 2991. Saline/dextrose was chosen as an appropriate re-suspension solution as the inclusion of dextrose aids metabolism, a point supported by increased ATP levels seen in these units in the current study. However, with a 24-hour outdate, use of a more complex re-suspension fluid (e.g. a RBC additive solution) was deemed unnecessary. This process change was validated and implemented at Canadian Blood Services, allowing the organization to meet the required Hct standard for deglycerolized RBC products.

Although these criteria do not apply in Canada, RCCs were also assessed against Council of Europe and AABB guidelines. Standards differ greatly from jurisdiction to jurisdiction, making comparison challenging. Cryopreservation procedures, which also differ among jurisdictions, affect the level of damage done to RBCs during the freeze/thaw process. For example, initial Hct levels, cryopreservation conditions, glycerol concentrations, and the stor- 
age solution after deglycerolization impact RBC recovery, hemolysis, and fragility post cryopreservation $[15,16]$. Thus it is unclear how well the results seen in this study with Canadian products and methods would translate to other products produced using different methods or with different instrumentation. It is worth nothing that in Europe, many are now using the ACP-215 closed system to wash thawed RCCs, and results would therefore expect to differ from those seen with the COBE 2991.

With the exception of the pilot phase, throughout the study CSA criteria for hemolysis were not met. Hemolysis represents terminal injury of the RBCs and is also a critical measure of RBC quality. Of the standards assessed in this study, only the CSA mandate hemolysis levels are measured. However, the Council of Europe stipulates supernatant $\mathrm{Hb}$ levels, and these were also not met at any stage during the study, including the pilot study. Like hemolysis, supernatant $\mathrm{Hb}$ levels are indicative of terminal damage to the RBCs. Together, the hemolysis and supernatant $\mathrm{Hb}$ levels suggest that the process of freezing and deglycerolization damages the RBCs. This is consistent with reports on the fragility of RBCs that are produced using the COBE 2991 [17]. Washed and deglycerolized RBC products produced on the COBE 2991 have an elevated level of hemolysis compared with unprocessed units, likely due to mechanical damage to the cells. In the original report by Meryman and Hornblower [17] in 1972 on the use of the COBE 2991 for the cryopreservation of RBCs, an almost 10-fold increase in supernatant $\mathrm{Hb}$ concentration following deglycerolization was reported, suggesting significant damage to the RBCs. Hemolysis during blood cell processing, including cell washing and deglycerolization has been well described $[10,18,19]$. Hemolysis levels in deglycerolized RBCs have been shown to be affected by many factors including RBC processing factors such as leukoreduction [20], collection and freezing containers [19], the additive solutions used post processing [16], age of the $\mathrm{RBC}$, and the specific methods used for glycerolization, freezing, thawing and cryopreservation $[4,15,21]$.

RBC hemolysis results observed in the pilot study were significantly lower $(\mathrm{p}<0.001)$ than the results observed in the validation study. The differences in hemolysis results between the pre- and post-implementation study compared to the pilot study show the limitations that can arise when testing moves from a controlled environment to a real-world situation. The pilot study had a small sample size and was a pool and split study, conducted in a completely controlled environment at a single site by two highly trained technicians. Also, different testing methods were used to measure hemolysis in the pilot study and the validation study, which could influence the results $[22,23]$. The pre- and post-implementation phase of this study was conducted at multiple production sites across Canada - seven sites in the pre-implementation portion and six in the post-implementation portion. Unlike the pilot study which was a pool and split study, donor factors and the impact of rare $\mathrm{RBC}$ phenotypes on $\mathrm{RBC}$ fragility may have been at play during the pre- and post-implementation phase. In the post-implementation phase, there may have been a learning curve with the new process and technician-to-technician differences in how the process was performed. Although designed to be simple, the new process does involve some manual steps where variances could appear, including the speed with which saline/dextrose solution is added post deglycerolization. Most importantly, the length of time before testing occurred may have affected hemolysis, as in both the validation and pre- and post-implementation studies, in line with QC requirements, testing may have occurred up to $48 \mathrm{~h}$ after deglycerolization (vs. maximal $24 \mathrm{~h}$ in the pilot study). Thus these hemolysis levels may not actually represent the level of hemolysis of the units at the time of transfusion ( $<24 \mathrm{~h}$ post deglycerolization).

Saline/dextrose was used as the re-suspension fluid due to its potential benefits for RBC quality versus saline alone. Addition of extra saline/dextrose led to an increase in ATP levels in the units. This is likely related to dextrose allowing maintenance of RBC metabolism [24], which is associated with better product quality. Lower levels of supernatant potassium were also seen in deglycerolized units to which additional saline/dextrose were added. Overall, despite the outdate being just $24 \mathrm{~h}$, the better maintenance of RBC metabolism and the lower dose of potassium per unit suggests improved RBC quality in the units to which re-suspension fluid was added. RBC quality indices did indicate an increase in MCV post deglycerolization in all groups, suggesting that swelling occurs with glycerolization/freezing/deglycerolization. The swelling observed due to the increased MCV could affect Hct results and create bias in other measurements that require this value (i.e., hemolysis, recovery, $\mathrm{Hb}$ /unit).

While all experimental groups passed the CSA guideline for $\mathrm{RBC}$ recovery, it is worth noting that the recovery calculation used in this study is limited. This calculation, which is used at Canadian Blood Services for QC purposes, assumes that the density of the pre- and post-deglycerolized products is the same. This assumption is not necessarily true, especially for the control units which have higher Hcts than pre-freeze units. This calculation also assumes that the RBC Hct is not affected pre and post deglycerolization and thus does not take into account swelling of the RBCs, which we clearly observed in this study. This study had several other limitations. Differences in the length of pre-freeze storage time, frozen storage time, and refrigerated storage post deglycerolization may impact comparisons across the pilot, validation and pre- and post-implementation studies. There were also differences in the ratio of buffy coat- and WB filtration-processed RCCs (and different bag set manufacturers) among the various phases of this study, and this may impact some of the results. In the validation study, the additional wash which was applied to some units - an additional manipulation that tends to increase hemolysis at expiry - may be a contributing factor to the levels of post-deglycerolization hemolysis at $24-48 \mathrm{~h}$. Similarly, for the pre- and post-implementation data it is not known whether the units received an additional wash, and thus we cannot conclude whether this was a contributing factor in the higher RBC hemolysis seen in these data.

The method explored in this study produced deglycerolized RCCs with Hct levels that were acceptable. Hct was the focus of this study as this standard was not being met, and it is an important measure that can impact how physicians treat patients with these products. With the original product - deglycerolized RBCs 
with a high Hct - physicians could infuse a higher number of RBC units due to the decrease in volume in comparison to a standard unit; thus delivering a higher dosage of RBCs to the recipient while avoiding circulatory overload. The units produced using the new method allow physicians to deliver a possibly better quality product due to the addition of extra dextrose in the supernatant; however, these products will have a lower RBC dosage overall because of the higher volumes of the units lead to fewer units that can be transfused. The difference in in vivo recovery is not known; however, these modified products have been available for use since 2013. Overall, deglycerolization with the COBE 2991 impacts RBC hemolysis, as seen in previous studies $[25,26]$. It is also worth noting that this technology produces products in an open system, and the resulting products are limited by a 24 -hour outdate. Despite being able to meet the Hct standard, the damage suggested by the hemolysis results in this study indicates that the COBE 2991 is not an ideal system for deglycerolizing RCCs. More modern, closedsystem technologies like the ACP-215 from Haemonetics Corporation permit the production of deglycerolized RCCs with an extended outdate time [16, 27-29]. These technologies are in use in many jurisdictions, have been implemented for RBC washing at Canadian Blood Services [30-33], and may provide a means to produce even higher-quality products with the added advantage of a longer outdate time.

\section{Acknowledgements}

The authors thank Caesar DiFranco, Canadian Blood Services QC manager for providing the pre- and post-implementation data; Della Reside, project manager, Michelle Rogerson, director, supply chain, and Terry Milne, validation associate who managed the validation study; Canadian Blood Services staff who performed the validation study in British Columbia and Yukon, Ottawa, New Brunswick, and Edmonton; Dr. Geraldine Walsh, Canadian Blood Services' scientific writer for assistance with manuscript preparation and editing; and all Canadian Blood Services donors.

\section{Disclosure Statement}

JPA has received grants from Canadian Blood Services, Canadian Institutes for Health Research, Natural Sciences and Engineering Research Council of Canada, and the Women and Children's Health Research Institute. He has received: honoraria as a member of BioLife Solutions' Scientific Advisory Board and HemoCue America LLC Advisory Board; consulting fees as a faculty member of Biomet Biologics' Education Team, and personal fees from Aquila Diagnostic Systems. TRT, ALH and JDRK have no conflicts of interest to disclose.

\section{References}

1 Lecak J, Scott K, Young C, Hannon J, Acker JP: Evaluation of red blood cells stored at -80 degrees $\mathrm{C}$ in excess of 10 years. Transfusion 2004;44:1306-1313.

2 Valeri CR, Ragno G, Pivacek LE, Cassidy GP, Srey R, Hansson-Wicher M, Leavy ME: An experiment with glycerol-frozen red blood cells stored at -80 degrees $\mathrm{C}$ for up to 37 years. Vox Sang 2000;79:168-174.

3 Henkelman S, Noorman F, Badloe JF, Lagerberg JW Utilization and quality of cryopreserved red blood cells in transfusion medicine. Vox Sang 2015;108:103-112.

4 Scott KL, Lecak J, Acker JP: Biopreservation of red blood cells: past, present, and future. Transfus Med Rev 2005; 19:127-142.

5 Canadian Standards Association: CAN/CSA-Z902-15 Blood and Blood Components. Toronto, Canadian Standards Association. 2015.

6 Council of Europe: Guide to the Preparation, Use and Quality Assurance of Blood Components, 18th ed. Strasbourg, Council of Europe Publishing, 2015.

7 AABB: Standards for Blood Banks and Transfusion Services. Bethesda, AABB, 2012.

8 Canadian Blood Services: Red Blood Cells, Leukocytes Reduced (LR), in Circular of Information for the use of Human Blood Components. , Ottawa, Canadian Blood Services, 2011.

9 Acker JP, Hansen AL, Kurach JD, Turner TR, Croteau I, Jenkins C: A quality monitoring program for red blood cell components: in vitro quality indicators before and after implementation of semiautomated processing. Transfusion 2014:54:2534-2543.

10 Sowemimo-Coker SO: Red blood cell hemolysis during processing. Transfus Med Rev 2002;16:46-60.

11 Keir AK, Hansen AL, Callum J, Jankov RP, Acker JP: Coinfusion of dextrose-containing fluids and red blood cells does not adversely affect in vitro red blood cell quality. Transfusion 2014;54:2068-2076.
12 Kenner T: The measurement of blood density and its meaning. Basic Res Cardiol 1989;84:111-124.

13 Mastronardi C, Perkins H, Derksen P, denAdmirant M, Ramirez-Arcos S: Evaluation of the BacT/ALERT 3D system for the implementation of in-house quality control sterility testing at Canadian Blood Services. Clin Chem Lab Med 2010;48:1179-1187.

14 Canadian Blood Services: Red Blood Cells, Leukocytes Reduced (LR). Circular of Information for the use of Human Blood Components, Ottawa, Canadian Blood Services, 2015. www.blood.ca/sites/default/files/RedBloodCellsLeukocytesReduced.pdf (last accessed October 17 2016).

15 Wagner CT, Burnett MB, Livesey SA, Connor J: Red blood cell stabilization reduces the effect of cell density on recovery following cryopreservation. Cryobiology 2000;41:178-194.

16 Valeri CR, Srey R, Tilahun D, Ragno G: The in vitro quality of red blood cells frozen with 40 percent (wt/ vol) glycerol at -80 degrees $C$ for 14 years, deglycerolized with the Haemonetics ACP 215, and stored at 4 degrees $\mathrm{C}$ in additive solution-1 or additive solution-3 for up to 3 weeks. Transfusion 2004;44:990-995.

17 Meryman HT, Hornblower M: A method for freezing and washing red blood cells using a high glycerol concentration. Transfusion 1972;12:145-156.

18 Harm SK, Raval JS, Cramer J, Waters JH, Yazer MH: Haemolysis and sublethal injury of RBCs after routine blood bank manipulations. Transfus Med 2012;22: 181-185.

19 Hornblower M, Meryman HT: Influence of the container material on the hemolysis of glycerolized red cells after freezing and thawing. Cryobiology 1974;11: 317-323.
20 Bohonek M, Petras M, Turek I, Urbanova J, Hradek T, Staroprazska V, Kostirova J, Horcickova D, Duchkova S: In vitro parameters of cryopreserved leucodepleted and non-leucodepleted red blood cells collected by apheresis or from whole blood and stored in AS-3 for 21 days after thawing. Blood Transfus 2014;12(suppl 1) s199-203.

21 Valeri CR, Pivacek LE, Cassidy GP, Ragno G: In vitro and in vivo measurements of human RBCs frozen with glycerol and subjected to various storage temperatures before deglycerolization and storage at 4 degrees $\mathrm{C}$ for 3 days. Transfusion 2001;41:401-405.

22 Han V, Serrano K, Devine DV: A comparative study of common techniques used to measure haemolysis in stored red cell concentrates. Vox Sang 2010;98:116-123.

23 Acker JP, Croteau I, Yi QL: An analysis of the bias in red blood cell hemolysis measurement using several analytical approaches. Clin Chim Acta 2012;413:17461752

24 Hess JR: An update on solutions for red cell storage. Vox Sang 2006;91:13-19.

25 O'Leary MF, Szklarski P, Klein TM, Young PP: Hemolysis of red blood cells after cell washing with different automated technologies: clinical implications in a neonatal cardiac surgery population. Transfusion 2011; 51:955-960.

26 Smith T, Riley W, Fitzgerald D: In vitro comparison of two different methods of cell washing. Perfusion 2013; 28:34-37.

27 Lelkens CC, de Korte D, Lagerberg JW: Prolonged post-thaw shelf life of red cells frozen without prefreeze removal of excess glycerol. Vox Sang 2015;108: 219-225. 
28 Valeri CR, Ragno G, Pivacek L, O’Neill EM: In vivo survival of apheresis RBCs, frozen with 40-percent (wt/ vol) glycerol, deglycerolized in the ACP 215, and stored at 4 degrees $\mathrm{C}$ in AS-3 for up to 21 days. Transfusion 2001;41:928-932.

29 Valeri CR, Ragno G, Pivacek LE, Srey R, Hess JR, Lippert LE, Mettille F, Fahie R, O’Neill EM, Szymanski IO: A multicenter study of in vitro and in vivo values in human RBCs frozen with 40-percent (wt/vol) glycerol and stored after deglycerolization for 15 days at 4 degrees $\mathrm{C}$ in AS-3:assessment of RBC processing in the ACP 215. Transfusion 2001;41:933-939.
30 Acker JP, Hansen AL, Yi QL, Sondi N, Cserti-Gazdewich C, Pendergrast J, Hannach B, Introduction of a closed-system cell processor for red blood cell washing: postimplementation monitoring of safety and efficacy. Transfusion 2016;56:49-57.

31 Hansen A, Yi QL, Acker JP: Quality of red blood cells washed using the ACP 215 cell processor: assessment of optimal pre- and post-wash storage times and conditions. Transfusion 2013;53:1772-1779.
32 Hansen AL, Turner TR, Kurach JD, Acker JP: Quality of red blood cells washed using a second wash sequence on an automated cell processor. Transfusion 2015;55:2415-2421.

33 Hansen AL, Turner TR, Yi QL, Acker JP: Quality of red blood cells washed using an automated cell processor with and without irradiation. Transfusion 2014;54: 1585-1594. 